\title{
Outcomes Following Use of a Modified Duran Ring Tricuspid Valve Reconstruction Procedure for Secondary Tricuspid Regurgitation
}

\author{
Sung-Ho Jung, MD; Hyung Gon Je, MD*; Jong Min Song, MD**; Suk Jung Choo, MD; \\ Cheol Hyun Chung, MD; Sung Cheol Yun, $\mathrm{PhD}^{\dagger}$; Jae Won Lee, MD
}

\begin{abstract}
Background: Tricuspid valve (TV) repair using ring annuloplasty is reportedly to be superior to suture annuloplasty for treating tricuspid regurgitation (TR). The Duran ring TV annuloplasty technique was modified in order to reduce the risk of complete atrioventricular block and aortic valve injury and the outcomes are reported here. The modification involved not suturing half of the septal annulus.

Methods and Results: Between January 1998 and July 2007, 219 patients diagnosed with secondary TR underwent TV repair using a modified Duran ring procedure. The mean patient age was $54.2 \pm 12.7$ years, and 65 (29.7\%) patients were male. The median follow-up duration was 35.8 months (range, 0.03-122.6 months). The mean ring size was $27.4 \pm 2.0 \mathrm{~mm}$. The prevalence of $3+$ or $4+$ regurgitation was $9.5 \%(21 / 218)$ at 1 week postoperatively, and $9.4 \%$ at more than 2 years postoperatively (mean, 57 months; 96 patients assessed). The in-hospital mortality rate was $1.4 \%(3 / 219)$. The overall survival rate was $95 \pm 1.5 \%$ at 1 year, $86.2 \pm 3.0 \%$ at 5 years, and $79.9 \pm 4.2 \%$ at 8 years. Multivariate analysis showed that atrial fibrillation at the last follow-up $(\mathrm{P}<0.001)$ and cardiopulmonary bypass time $(\mathrm{P}=0.016)$ were risk factors for recurrent or persistent significant TR.
\end{abstract}

Conclusions: The modified Duran ring TV repair procedure for secondary TR patients was safe and durable. Notably, postoperative atrial fibrillation was found to be a significant risk factor for recurrent TR. (Circ J 2010; 74: 925-930)

Key Words: Annuloplasty; Atrial fibrillation; Duran ring; Recurrence; Tricuspid regurgitation

$\mathbf{T}$ ricuspid regurgitation (TR) is commonly caused by pressure or volume overloading because of left-side heart disease or congenital heart disease. In TR, the tricuspid valve (TV) annulus is dilated mainly in the anterior and posterior leaflet portions. ${ }^{1}$ There are many surgical techniques for tricuspid annuloplasty (TAP) used to reduce the annular diameter in TR patients. Some reports conclude that ring annuloplasty is superior to DeVega-type annuloplasty. ${ }^{2,3}$ For ring annuloplasty, rings can either flexible or rigid. Although a recent report described the use of a rigid 3-dimensional (D) ring, ${ }^{4}$ variations in TV shape during the cardiac cycle suggest a flexible ring would be better. ${ }^{5}$ Duran et al described TAP using a flexible ring (Duran ring) in which they sutured the annulus completely. ${ }^{6,7}$ However, this approach carries the risk of injury to conduction tissue and the aortic valve.
We describe our experience with using a modified method of Duran ring TAP for secondary TR. The modification involves suturing only half of the septal annulus in order to reduce the risk of complete atrioventricular block and aortic valve injury.

Methods
Patients
Application of a Duran ring to the TV was used from 1998.
From March 1998 to June 2007, a total of 219 consecutive
patients diagnosed with secondary TR underwent Duran ring
annuloplasty [65 (30\%) males, 154 females; age range, 22-83
years (mean, 54.2 years)]. To obtain a uniform study group,
patients with isolated TR or primary pathologic change of
the TV were excluded because they frequently require more

Received November 2, 2009; revised manuscript received December 27, 2009; accepted January 12, 2010; released online March 26, 2010 Time for primary review: 21 days

Department of Thoracic and Cardiovascular Surgery, Asan Medical Center, College of Medicine, University of Ulsan, Seoul, *Department of Cardiovascular Surgery, Pusan National University Yangsan Hospital, Yangsan, **Department of Cardiology, Asan Medical Center, College of Medicine, University of Ulsan and 'Department of Preventive Medicine, Asan Medical Center, College of Medicine, University of Ulsan, Seoul, Republic of Korea

Mailing address: Jae Won Lee, MD, PhD, Department of Thoracic and Cardiovascular Surgery, Asan Medical Center, 388-1 PoongnapDong, Songpa-Ku, Seoul 138-736, Republic of Korea. E-mail: jwlee@ amc.seoul.kr

ISSN-1346-9843 doi:10.1253/circj.CJ-09-0845

All rights are reserved to the Japanese Circulation Society. For permissions, please e-mail: cj@j-circ.or.jp 


\begin{tabular}{|lc|}
\hline \multicolumn{2}{l}{ Table 1. Preoperative Demographic and Hemodynamic Data } \\
Age (years) & $54.2 \pm 12.7$ \\
Sex (Male) & $65(29.7 \%)$ \\
LVEF (\%) & $55.9 \pm 10.3$ \\
AF & $180(82.2 \%)$ \\
NYHA functional class & \\
I & $12(5.5 \%)$ \\
II & $99(45.2 \%)$ \\
III & $91(41.5 \%)$ \\
IV & $17(7.8 \%)$ \\
Pulmonary hypertension* & $97(44.1 \%)$ \\
TR & \\
I & $2(0.9 \%)$ \\
II & $28(12.8 \%)$ \\
III & $75(34.2 \%)$ \\
IV & $113(51.6 \%)$ \\
\hline
\end{tabular}

*Pulmonary hypertension: ESRVP $>50 \mathrm{mmHg}$.

LVEF, left ventricular ejection fraction; $A F$, atrial fibrillation; NYHA, New York Heart Association; TR, tricuspid regurgitation; ESRVP, estimated systolic right ventricular pressure.

than simple annuloplasty. Twelve patients (5.5\%) were in New York Heart Association (NYHA) functional class I, 99 $(45.2 \%)$ in class II, $91(41.6 \%)$ in class III, and $17(7.8 \%)$ in class IV. A total of 180 patients $(82.2 \%)$ had atrial fibrillation (AF) and 198 (90\%) patients had combined mitral valve disease (Table 1).

Prior cardiac surgery had been performed in 25 (11.4\%) patients. Reasons for reoperation were previous valve repair failure $(n=9)$, previous prosthetic valve failure $(n=6)$, infec- tive endocarditis $(n=3)$, progression of other valve disease $(\mathrm{n}=3)$, and postoperative complications such as paravalvular leakage $(n=1)$, constrictive pericarditis $(n=1)$, and post tetralogy of Fallot $(n=1)$. Of these reoperation patients, previous TV repair (TVR) had been performed in 5 patients, comprising 2 patients who had a Carpentier-Edwards ring inserted and 3 patients who underwent a Kay procedure; 18 patients $(8.2 \%)$ had undergone prior percutaneous mitral valvuloplasty. TR severity was assessed using color Doppler flow criteria $^{8}$ and graded as: 0 (no TR), 1 (mild TR), 2 (mild to moderate TR), 3 (moderate to severe TR), or 4 (severe) by routine clinical assessment of the visually estimated size of the regurgitant area seen with color Doppler echocardiography.

To determine the risk factors for remnant significant TR (TR 3+ or 4+), we defined pulmonary arterial hypertension as an estimated right ventricular systolic pressure $>50 \mathrm{mmHg}$ on echocardiographic examination. Right ventricular systolic pressures were estimated using continuous-wave Doppler data and the simplified Bernoulli equation [ $\left.4 \times(\text { peak TR velocity })^{2}\right]$ with $10 \mathrm{mmHg}$ added for the estimated right atrial pressure. ${ }^{9}$

All surgery was performed by the same surgeon. Followup information was obtained from medical records. If the patient had not been seen in the clinic within the previous 6 months, follow-up information was obtained from telephone conversations between the patient and an investigator. When it was impossible to contact patients, we referred to the National Health Insurance Corporation database to determine whether they were still living. The median follow-up duration was 35.8 months (range, 0.03-122.6 months).

\section{Decision for TVR}

Clinical signs of right heart failure and moderate to severe TR according to preoperative cardiac echocardiography are recognized indications for tricuspid insufficiency repair at the

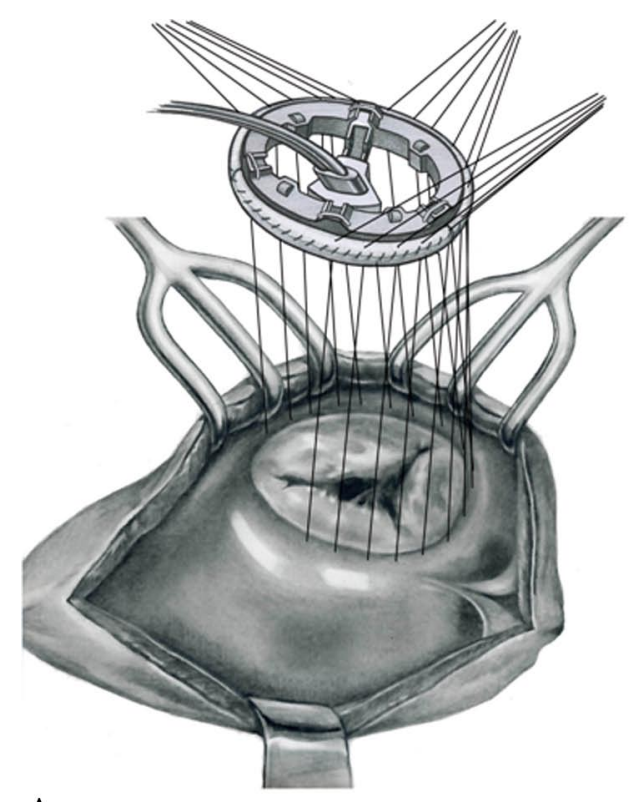

A

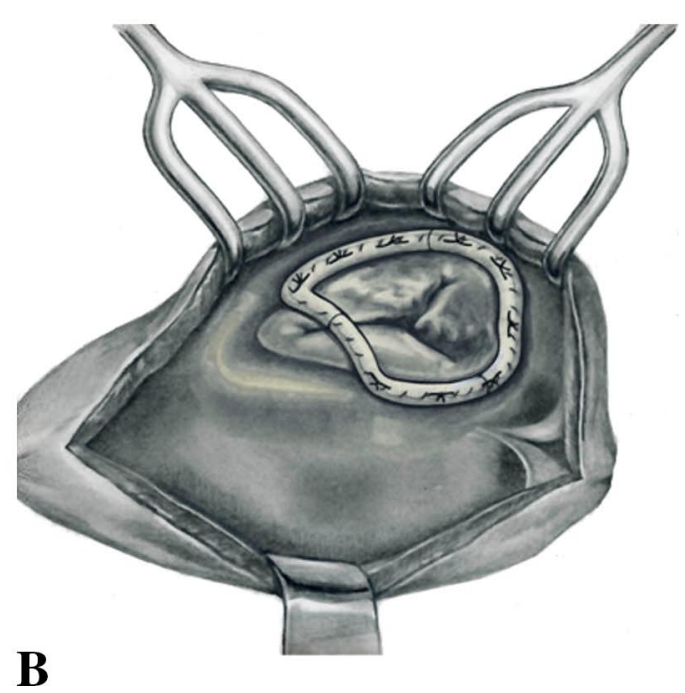

Figure 1. (A) Annuloplasty sutures placed along the tricuspid annulus except for the medial half of the septal annulus and the anterior annulus beyond the aortic prominence. (B) Partial tricuspid annuloplasty using a flexible ring allows the annulus to remain flexible while preserving the orifice area and optimizing leaflet coaptation. 
time of replacement or repair of left-sided heart valves. TAP was performed in patients with a huge right atrium or a dilated tricuspid annulus even if the TR was less than grade $2 .{ }^{1}$

\section{Surgical Technique}

Cardiopulmonary bypass (CPB) was performed using bicaval venous cannulation and correction of left-side lesions or other disease was done first. After the right atrium was opened, ring size was decided based on anterior leaflet size. A counterclockwise U stitch with 2-0 multifilament suture was performed from the center of the septal annulus through the anterior annulus to the aortic prominence. The risk of injury to the conduction system was prevented by avoiding the clockwise direction suture at the septal annulus (Figures 1A,B). A narrow stitch was placed at both the septal and posterior annulus to provide a Kay annuloplasty-like effect. Transesophageal echocardiography was performed to assess postoperative TR or stenosis.

\section{Statistical Analysis}

Categorical variables are presented as numbers and percentages, and were compared using the chi-square test. Continuous variables are expressed as mean $\pm \mathrm{SD}$ or median (range), and were compared using the Student's unpaired t-test. The overall survival curves were constructed with Kaplan-Meier method. Univariate and multivariate Cox proportional hazards models were used to examine the risk factor for recurrent or persistent significant TR. Multivariate analyses involved a backward elimination technique and only variables with a P-value $<0.10$ were used in the final model. All reported $\mathrm{P}$-values were 2 -sided, and a $\mathrm{P}$-value $<0.05$ was considered statistically significant. SPSS version 14.0 (Korean version; SPSS, Inc, Chicago, IL, USA) was used for statistical analyses.

\section{Results}

Mitral valve procedures including repair or replacement were performed in $198(90 \%)$ patients. Other concomitant procedures are summarized in Table 2. The mean CPB time and aorta clamp time was $156.3 \pm 52.7 \mathrm{~min}$ and $105.0 \pm 31.1 \mathrm{~min}$, respectively. The mean ring size was $27.4 \pm 2.0(25-35) \mathrm{mm}$. Thirty three patients underwent minimally invasive surgery using AESOP 3000 for mitral valve repair (MVR; $n=16$ ), mitral valve replacement $(n=16)$, a Maze operation $(n=23)$, and atrial septal defect patch closure $(n=5)$.

\section{In-Hospital Mortality and Complications, Reoperations, and Functional Improvement}

There were 3 in-hospital deaths $(3 / 219 ; 1.4 \%)$. One patient died from left ventricular rupture after undergoing MVR (Carpentier-Edwards Bioprosthetic valve $27 \mathrm{~mm}$ ), TAP

\begin{tabular}{|lc|}
\hline \multicolumn{1}{|c|}{$\begin{array}{c}\text { Table 2. Concomitant Procedures and Operative Data } \\
\text { Procedure }\end{array}$} & $\mathbf{n}=\mathbf{2 1 9}$ \\
AVR/AVP & $39(17.8 \%) / 1(0.5 \%)$ \\
MVR/MVP & $110(50.2 \%) / 88(40.2 \%)$ \\
Maze operation & $141(64.4 \%)$ \\
ASD closure & $17(7.7 \%)$ \\
VSD closure & $4(1.8 \%)$ \\
Pulmonary valve replacement & $3(1.4 \%)$ \\
Coronary artery bypass surgery & $14(6.4 \%)$ \\
Right minithoracotomy approach & $33(15.1 \%)$ \\
Duran ring size (mm) & $27.4 \pm 2.0$ \\
25 & $51(23.3 \%)$ \\
27 & $104(47.5 \%)$ \\
29 & $42(19.2 \%)$ \\
31 & $16(7.3 \%)$ \\
33 & $5(2.3 \%)$ \\
35 & $1(0.5 \%)$ \\
ACC time (min) & $105.0 \pm 33.1$ \\
CPB time (min) & $156.3 \pm 52.7$ \\
\hline
\end{tabular}

AVR, aortic valve replacement; AVP, aortic valvuloplasty; MVR, mitral valve replacement; MVP, mitral valvuloplasty; ASD, atrial septal defect; VSD, ventricular septal defect; ACC, aorta crossclamp; CPB, cardiopulmonary bypass time.

(Duran ring $27 \mathrm{~mm}$ ), and a Maze operation (cryocath) using a minithoracotomy approach. The second patient had sudden cardiac arrest possibly because of ventricular arrhythmia after MVR, TAP, and a Maze operation. The third patient underwent MVP, TAP and a Maze operation for dilated cardiomyopathy, mitral regurgitation, TR, and AF and showed low cardiac output syndrome postoperatively. Postoperative complications occurred in 25 patients and comprised postoperative bleeding requiring re-exploration $(n=12)$, acute renal failure $(n=4)$, pericardial effusion requiring pericardiocentesis $(n=3)$, seizure $(n=1)$, right ventricular assist device insertion $(n=1)$, delayed sternal closure $(n=1)$, sternal wound infection or dehiscence $(n=4)$, and esophageal ulcer $(n=1)$. Postoperative permanent pacemaker insertion was done in $6(2.7 \%)$ patients, among whom 4 underwent a Maze operation for AF.

Four patients underwent further surgery during the followup period. Only $1(0.5 \%)$ of these procedures was a reoperative TVR performed 18 months after the initial surgery. The surgical findings were disruption of 1 annular stitch, and therefore we obliterated the space with another stitch, which resulted in no further regurgitation during the follow-up period. The mean NYHA functional class score significantly improved from 2.5 to 1.1. There were 21 late deaths, including 4 patients with malignancy. The overall survival rate

\begin{tabular}{|lccccccc|}
\hline \multicolumn{7}{|c|}{ Table 3. Postoperative Changes in TR Over the Follow-up Period } \\
& $\begin{array}{c}\text { Pre-op } \\
(\mathbf{n = 2 1 8})\end{array}$ & $\begin{array}{c}\mathbf{1} \text { week } \\
(\mathbf{n = 2 1 7})\end{array}$ & $\begin{array}{c}\mathbf{3} \text { months } \\
(\mathbf{n = 1 2 4})\end{array}$ & $\begin{array}{c}\mathbf{6} \text { months } \\
(\mathbf{n = 1 2 3})\end{array}$ & $\begin{array}{c}\mathbf{1} \text { year } \\
(\mathbf{n = 1 3 4 )}\end{array}$ & $\begin{array}{c}\mathbf{2} \text { years } \\
(\mathbf{n = 1 0 4 )}\end{array}$ & $\begin{array}{c}\text { Last } \\
(\mathbf{n = 9 6 )}\end{array}$ \\
TR 0 & 0 & 43 & 21 & 28 & 25 & 22 & 33 \\
TR 1 & 2 & 106 & 75 & 72 & 81 & 64 & 40 \\
TR 2 & 28 & 47 & 11 & 17 & 19 & 9 & 14 \\
TR 3 & 75 & $15(6.9 \%)$ & $14(11.3 \%)$ & $3(2.4 \%)$ & $5(3.7 \%)$ & $7(6.7 \%)$ & $6(6.3 \%)$ \\
TR 4 & 113 & $6(2.8 \%)$ & $3(2.4 \%)$ & $3(2.4 \%)$ & $4(3.0 \%)$ & $2(1.9 \%)$ & $3(3.1 \%)$ \\
Significant TR & & $21(9.6 \%)$ & $17(13.7 \%)$ & $6(4.8 \%)$ & $9(6.7 \%)$ & $9(8.6 \%)$ & $9(9.4 \%)$ \\
\hline
\end{tabular}

Abbreviation see in Table 1. 


\begin{tabular}{|c|c|c|c|}
\hline & $\begin{array}{l}\text { TR aggravated group } \\
\qquad(n=26)\end{array}$ & $\begin{array}{l}\text { TR non-aggravated group } \\
\qquad(\mathrm{n}=191)\end{array}$ & $P$ value \\
\hline Age (years) & $58.9 \pm 11.8$ & $53.4 \pm 12.7$ & 0.038 \\
\hline LVEF (\%) & $51.2 \pm 10.7$ & $56.6 \pm 10.0$ & 0.011 \\
\hline Ring size $(\mathrm{mm})$ & $27.6 \pm 1.9$ & $27.4 \pm 2.0$ & 0.546 \\
\hline AF (preoperation) & $24(92.3 \%)$ & $154(80.6 \%)$ & 0.281 \\
\hline Maze operation & $15(57.7 \%)$ & $124(64.9 \%)$ & 0.516 \\
\hline AF (last rhythm in $<6$ months) & $13 / 25(52.0 \%)$ & $49 / 181(27.1 \%)$ & 0.018 \\
\hline Preoperative TR grade & $3.54 \pm 0.65$ & $3.35 \pm 0.75$ & 0.219 \\
\hline ESRVP >50 mmHg & $13(50.0 \%)$ & $83(42.9 \%)$ & 0.675 \\
\hline
\end{tabular}

Abbreviations see in Tables 1,2.

\begin{tabular}{|c|c|c|c|c|}
\hline & \multicolumn{2}{|c|}{ Univariate analysis } & \multicolumn{2}{|c|}{ Multivariate analysis } \\
\hline & HR & P-value & HR & P-value \\
\hline Age & 1.031 & 0.081 & & \\
\hline CPB time & 1.006 & 0.014 & 1.005 & 0.016 \\
\hline Ring size & 1.111 & 0.263 & & \\
\hline Preoperative AF & 4.873 & 0.121 & & \\
\hline Last FU AF & 4.637 & $<0.001$ & 4.614 & $<0.001$ \\
\hline Maze operation & 0.528 & 0.110 & & \\
\hline ESRVP $>50 \mathrm{mmHg}$ & 1.009 & 0.477 & & \\
\hline
\end{tabular}

$\mathrm{HR}$, hazard ratio; FU, follow up. Other abbreviations see in Tables 1 , 2.

was $95 \pm 1.5 \%$ at 1 year, $86.2 \pm 3.0 \%$ at 5 years, and $79.9 \pm 4.2 \%$ at 8 years.

\section{Changes in TR Over Time}

Immediate postoperative echocardiograms performed at 1 week after operation showed rapid recovery of regurgitation. The prevalence of $3+$ or 4+ regurgitation was 9.7\% (21/217) at 1 week postoperatively. Echocardiographic follow-up at $>2$ years postoperatively was possible in 96 patients, and showed that the prevalence of $3+$ or $4+$ regurgitation was $9.4 \%$ at $57 \pm 19.5$ months postoperatively (Table 3 ). These data indicate that the level of regurgitation remained stable over time. The main mechanism of TR in the early and late periods was incomplete coaptation with or without the tethering of the TV leaflet and the uncovered part with the ring was not involved in the mechanism of postoperative significant TR.

\section{Risk Factors for Postoperative Significant TR}

During the early postoperative period ( $<6$ months), significant TR was found to be more prevalent in patients of older age, with a low ejection fraction, or with postoperative AF (Table 4).

Univariate and multivariate analyses showed that CPB time and $\mathrm{AF}$ at the last follow-up were risk factors for persistent or recurrent TR (Table 5).

\section{D Echocardiogram of the TV After Ring Annuloplasty}

Changes in the shape of the flexible Duran ring during the cardiac cycle were confirmed using 3D echocardiograms (Figure 2). The ring opening was smaller during the systolic phase and larger during the diastolic phase.

\section{Discussion}

The most common presentation of TR is secondary to cardiac valvular pathology on the left side of the heart. Pressure overloading because of left-side heart valve disease or volume
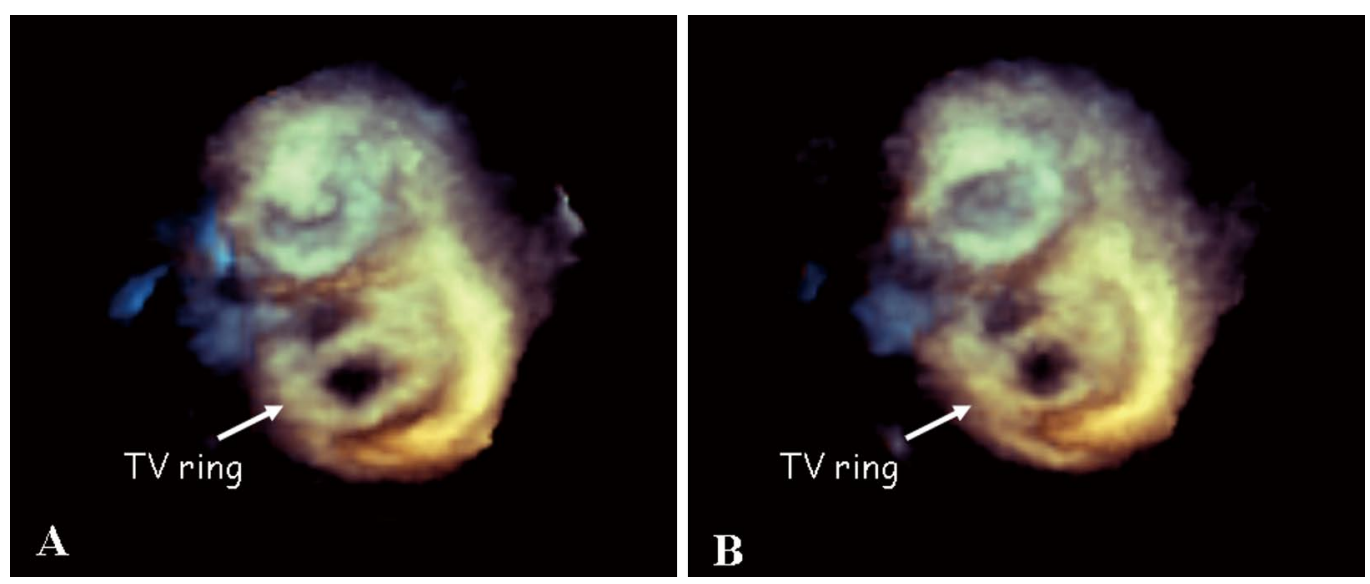

Figure 2. Three-dimensional echocardiographic findings at 6 months postoperatively. (A) Diastolic phase, (B) systolic phase. TV, tricuspid valve. 
overloading because of congenital heart disease, such as an atrial septal defect, causes the right ventricular dilatation resulting in dilation of the TV annulus. In the past, TR was not considered an important pathologic finding as it was not associated with significant symptoms and was believed to improve when the underlying cause(s) was treated. ${ }^{10}$ However, as with mitral regurgitation, it was found that severe TR was associated with a poor prognosis. ${ }^{11}$

Techniques for treating a dilated TV annulus with normal leaflets and chordal structures include plication of the posterior leaflet annulus (bicuspidization), partial purse-string reduction of both the anterior and posterior leaflet annulus (DeVega-style techniques), and rigid or flexible rings or bands placed to reduce the annular size and achieve leaflet coaptation.

Tsakiris et al showed that the normal tricuspid annulus undergoes continuous changes in size and shape over the cardiac cycle, ${ }^{5}$ which resulted in the development of the completely flexible Duran prosthetic ring that follows rather than interferes with these movements. ${ }^{7}$ The tricuspid annulus is nonplanar and saddle-like shape during systole, and more planar during diastole. It is somewhat triangular in shape, with a flatter septal side. The nonplanarity (saddle shape), excursion and change in area are preserved in patients that have undergone tricuspid valvuloplasty using a flexible ring. In their initial study, Duran et al described complete annulus suturing when using the Duran ring for TAP. ${ }^{7}$ However, we believe that this complete suture technique may carry the risk of injury to the conduction system and aortic mount. Therefore, we modified the technique such that half of the septal annulus adjacent to these structures is not sutured. The advantages of this modified approach are that it avoids injuring the conduction system, the ring is flexible such that heart motion and movement are conserved, it prevents injury to the aortic mount, allows for selective annulus remodeling at the posteroseptal area, and improves coaptation because of anteroposterior dimension reduction. We used the total ring instead of a band in order to preserve the acute angle of the anteroseptal commissure and keep the shape of the original TV more effectively. The possible disadvantages are hemolysis related to the ring itself, para-ring leakage, and that the procedure is more time-consuming compared with DeVegaor Kay-type surgery. However, in the present study no patients experienced para-ring leakage or hemolysis because of the ring, and we believe that the superior long-term results outweigh the disadvantage of increasing the surgery time by approximately $15 \mathrm{~min}$ in order to perform ring annuloplasty. Recently, Filsoufi et al reported on results following annuloplasty using the Edwards $\mathrm{MC}^{3}$ system. ${ }^{4}$ They emphasized the 3D structure of the TV and 3D reconstruction of TR. However, that particular ring is rigid and similar to the traditional Carpentier-Edwards tricuspid ring in terms of tricuspid annulus changes during the cardiac cycle.

Some studies have found that the mean rate of freedom from tricuspid repair failure (ie, clinical right heart failure, redo annuloplasty, and TV replacement at follow-up) was similar regardless of the annuloplasty technique used. ${ }^{12}$ Meanwhile, others reported that ring annuloplasty is superior to DeVega-type annuloplasty in terms of residual TR., ${ }^{2,3}$ In the present series, only 1 patient required reoperation because of postoperative significant TR. Freedom from reoperation in our study population was excellent but the incidence of significant TR was similar to other reports. ${ }^{2}$

The hospital mortality rate in our series was $1.4 \%$, which is superior to rates of 5.3-28\% reported in other similar series.
We observed a higher incidence of postoperative bleeding than reported elsewhere, and this may reflect the preoperative hepatomegaly and coagulopathy in patients with significant TR. Postoperative permanent pacemaker insertion was done in $6(2.7 \%)$ patients and the incidence of permanent pacemaker insertion is low, considering the incidence of permanent pacemaker insertion with the Maze operation. ${ }^{13}$ However, 2 of 6 patients did not undergo the Maze operation and the permanent pacemaker insertion in these patients was because of tachybradycardia syndrome with sinus pause and late-onset (3 years) complete atrioventricular block. We do not consider that these cases were related to the ring annuloplasty.

In the present study, also, early postoperative TR was 3+ or $4+$ in $11-13 \%$ and remained stable over time at less than $10 \%$ (Table 3). These outcomes are similar to others showing a residual TR rate of $14 \%$ in the early postoperative period for all types of annuloplasty, ${ }^{2}$ and that regurgitation severity remained stable over time when the CarpentierEdwards ring was used. However, Filsoufi et al reported excellent results using the $3 \mathrm{D}$ rigid ring and predischarge echocardiography did not show any significant TR. ${ }^{4}$ They emphasized remodeling annuloplasty using a small size ring. In the present study, however, a size $25 \mathrm{~mm}$ or $27 \mathrm{~mm}$ was used in $71 \%$ of patients, and rings that were greater than or equal to $31 \mathrm{~mm}$ were implanted in only $10 \%$ of patients. Thus the use of small-sized ring did not differ from their report. Moreover, the ring size was not a significant risk factor for recurrent or persistent significant TR in our series. Further study will be necessary to compare the direct results according to ring type.

We previously reported that using the Duran ring in the mitral valve may be the cause of mitral stenosis from pannus formation. ${ }^{14}$ However, that scenario is not relevant when using the ring in the TV, because of the different anatomical relationship between the inlet and outlet positions. We found that no patients developed tricuspid stenosis because of pannus formation.

Multivariate analysis showed that the presence of AF at the last follow-up was a risk factor for recurrent TR. Preoperative AF or the Maze operation were not significant factors, indicating that postoperative normal sinus rhythm is an important factor in successful treatment of TR. More aggressive surgery for AF in patients with TR is recommended. The CPB time was another risk factor for recurrent TR, which might be related to complex surgery such as redo operations. In the present study, the main mechanism for recurrent or persistent TR was incomplete coaptation with or without the tethering effect of the TV leaflet. We believe this is related to RV dysfunction and remodeling. ${ }^{15}$ Complex surgery needing a longer CPB time might cause postoperative RV dysfunction and we consider that a longer CPB time became a risk factor in the present study for this reason. However, we did not measure RV function quantitatively, so we could not correlate significant TR with RV dysfunction in the present study.

The TV is not a passive structure but rather forms a dynamic part of the right ventricle. ${ }^{16}$ Its orifice area changes not only because of contraction and expansion of its perimeter but also because of changes in its saddle shape. Recently, 3D echocardiography was developed for assessing TV morphology, ${ }^{17}$ so in the present study, we could confirm such shape changes according to the phase of the cardiac cycle. 


\section{Study Limitations}

First, the study did not include a non-ring annuloplasty control group. In our institute, there is a tendency to use ring annuloplasty in patients with more severe tricuspid annulus dilatation or a more dilated right atrium. Therefore, comparisons between ring annuloplasty and non-ring annuloplasty patients would have been compromised as the 2 techniques tend to be used on different patient groups. Second, the echocardiographic data obtained were subject to significant selection and ascertainment bias. Symptomatic patients were probably more likely to receive follow-up echocardiography than asymptomatic patients. The true TR prevalence may in fact have been lower if all patients had routinely undergone echocardiography screening. Third, this is a retrospective review, and thus contains all the inherent limitations of such a study design. However, all surgery was conducted by a single surgeon, and almost all patients underwent Duran ring annuloplasty during the study period. Therefore, we believe that selection bias and technical variation would be minimal despite the retrospective single-center nature of the study.

\section{Conclusion}

The present study found that a modified TV reconstruction procedure using a Duran ring, in which half of the septal annulus was not sutured, was safe and durable. Notably, postoperative AF was found to be a significant risk factor for recurrent TR.

\section{References}

1. Dreyfus GD, Corbi PJ, Chan KM, Bahrami T. Secondary tricuspid regurgitation or dilatation: Which should be the criteria for surgical repair? Ann Thorac Surg 2005; 79: 127-132.

2. McCarthy PM, Bhudia SK, Rajeswaran J, Hoercher KJ, Lytle BW, Cosgrove DM, et al. Tricuspid valve repair: Durability and risk factors for failure. J Thorac Cardiovasc Surg 2004; 127: 674-685.

3. Tang GH, David TE, Singh SK, Maganti MD, Armstrong S, Borger MA. Tricuspid valve repair with an annuloplasty ring results in improved long-term outcomes. Circulation 2006; 114(Suppl): I-577-
I-581.

4. Filsoufi F, Salzberg SP, Coutu M, Adams DH. A three-dimensional ring annuloplasty for the treatment of tricuspid regurgitation. Ann Thorac Surg 2006; 81: 2273-2277.

5. Tsakiris AG, Mair DD, Seki S, Titus JL, Wood EH. Motion of the tricuspid valve annulus in anesthetized intact dogs. Circ Res 1975; 36: $43-48$.

6. Duran CG, Ubago JL. Clinical and hemodynamic performance of a totally flexible prosthetic ring for atrioventricular valve reconstruction. Ann Thorac Surg 1976; 22: 458-463.

7. Duran CMG. Duran ring annuloplasty of the tricuspid valve. Oper Tech Thorac Cardiovasc Surg 2003; 8: 201-212.

8. Gonzalez-Vilchez F, Zarauza J, Vazquez de Prada JA, Martin Duran R, Ruano J, Delgado C, et al. Assessment of tricuspid regurgitation by Doppler color flow imaging: Angiographic correlation. Int J Cardiol 1994; 44: 275-283.

9. Currie PJ, Seward JB, Chan KL, Fyfe DA, Hagler DJ, Mair DD, et al. Continuous wave Doppler determination of right ventricular pressure: A simultaneous Doppler-catheterization study in $127 \mathrm{pa}-$ tients. J Am Coll Cardiol 1985; 6: 750-756.

10. Shah PM, Raney AA. Tricuspid valve disease. Curr Prob Cardiol 2008; 33: 47-84.

11. Nath J, Foster E, Heidenreich PA. Impact of tricuspid regurgitation on long-term survival. J Am Coll Cardiol 2004; 43: 405-409.

12. Carrier M, Pellerin M, Guertin MC, Bouchard D, Hebert Y, Perrault LP, et al. Twenty-five years' clinical experience with repair of tricuspid insufficiency. J Heart Valve Dis 2004; 13: 952-956.

13. Gaynor SL, Schuessler RB, Bailey MS, Ishii Y, Boineau JP, Gleva MJ, et al. Surgical treatment of atrial fibrillation: Predictors of late recurrence. J Thorac Cardiovasc Surg 2005; 129: 104-111.

14. Chung CH, Kim JB, Choo SJ, Kim KS, Song H, Song MG, et al. Long-term outcomes after mitral ring annuloplasty for degenerative mitral regurgitation: Duran ring versus Carpentier-Edwards ring. J Heart Valve Dis 2007; 16: 536-544; discussion 44-45.

15. Seo HS, Ha JW, Moon JY, Choi EY, Rim SJ, Jang Y, et al. Right ventricular remodeling and dysfunction with subsequent annular dilatation and tethering as a mechanism of isolated tricuspid regurgitation. Circ J 2008; 72: 1645-1649.

16. Jouan J, Pagel MR, Hiro ME, Lim KH, Lansac E, Duran CM. Further information from a sonometric study of the normal tricuspid valve annulus in sheep: Geometric changes during the cardiac cycle. J Heart Valve Dis 2007; 16: 511-518.

17. Seo Y, Ishizu T, Nakajima H, Sekiguchi Y, Watanabe S, Aonuma K. Clinical utility of 3-dimensional echocardiography in the evaluation of tricuspid regurgitation caused by pacemaker leads. Circ $J$ 2008; 72: $1465-1470$. 